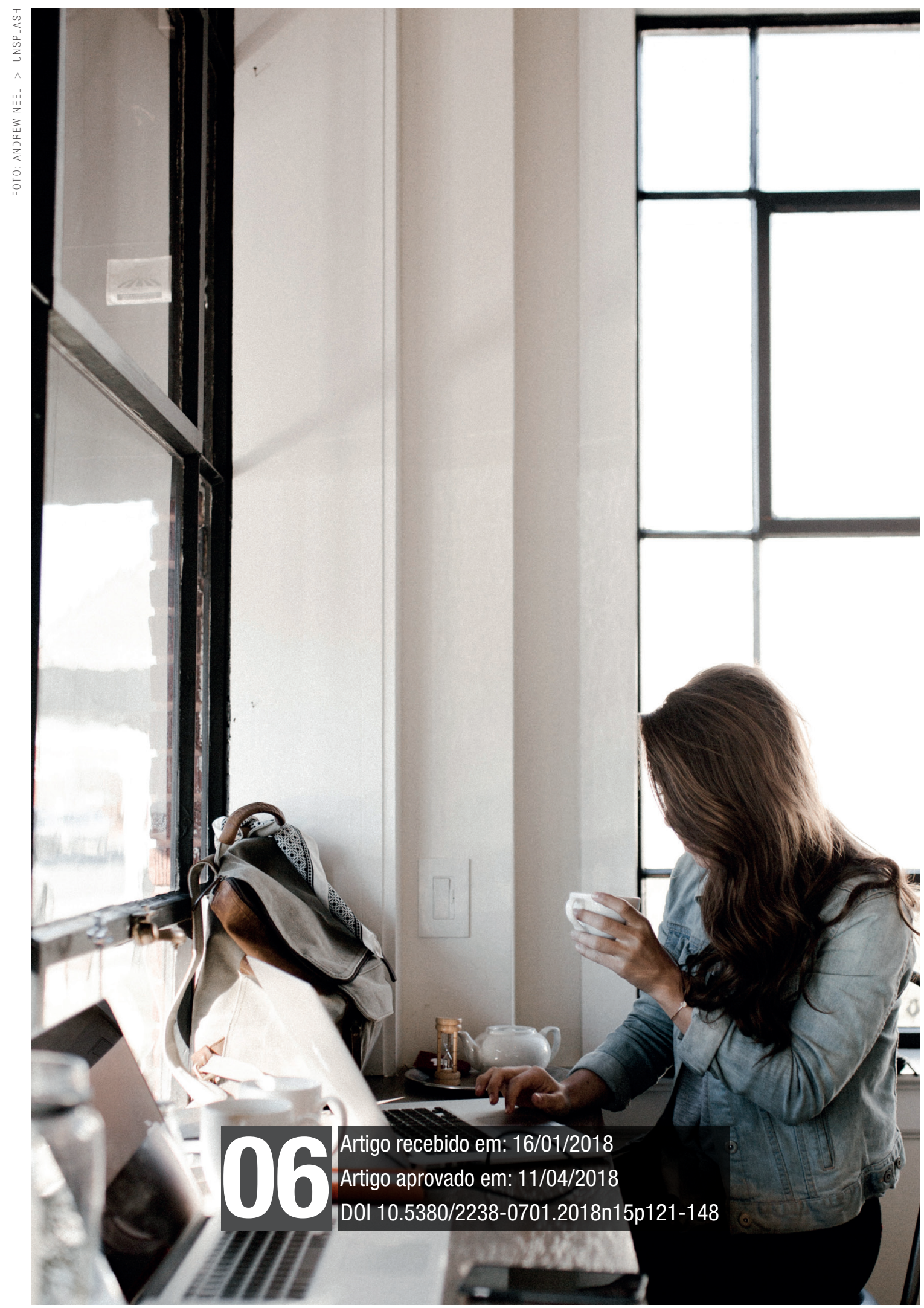


Financiamento, meios de comunicação, media, jornalismo, crowdfunding, Líbano. 


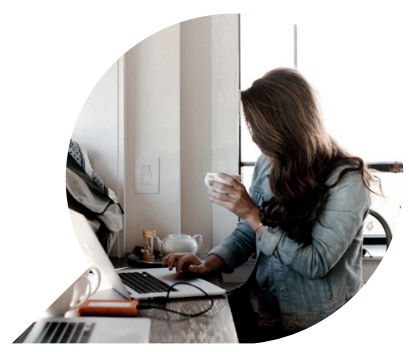

\section{Le financement participatif des médias: une exploration des perceptions des journalistes et des étudiants en journalisme au Liban}

Crowdfunding the media: an exploratory study of journalists and journalism students' perceptions in Lebanon

RAYAN HAYKAL ${ }^{1}$

JENNY MCHANTAF ${ }^{2}$

Résumé: Les difficultés financières que connaît le monde médiatique poussent à la recherche de nouveaux modes de financement qui assureraient la soutenabilité du média et conserveraient la liberté de son expression. Le financement participatif (Crowdfunding) a déjà été utilisé de manière efficace dans certains domaines artistiques et s'inscrit dans une logique d'éclatement des modèles économiques traditionnels. Une enquête est menée auprès des journalistes et des étudiants en journalisme au Liban pour sonder leurs perceptions de ce mode de financement alternatif et de ce qu'il leur représente. Les résultats de cette étude exploratoire montrent que le phénomène est encore mal connu au Liban et suscite peu l'enthousiasme d'un public bien spécifique, et concernerait des médias bien ciblés.

1 Coordenador do Departamento de Economia da Sagesse University.

2 Doutora em Media Studies pela Université Catholique de Louvain, professora assistente no Centro de Educação Continuada da Antonine University. 
Abstract: Financial distress in the media world is pushing forward to finding new funding tools in order to ensure media's sustainability without compromising freedom of expression. Crowdfunding has proven its efficiency in funding artistic projects and helps in breaking up traditional business models. A survey is conducted among journalists and journalism students in Lebanon to investigate their perceptions of this alternative form of financing. The results of this exploratory study show that the phenomenon is still poorly known in Lebanon and does not arouse the enthusiasm of one specific public, and would only concern some targeted media.

Resumo: As dificuldades financeiras sofridas pelos meios de comunicação levam as organizações a explorar novas fontes de receita que lhes permitam assegurar a sustentabilidade das respetivas atividades sem comprometer a liberdade de imprensa. O crowdfunding já demonstrou ser uma ferramenta eficiente no financiamento de projetos artísticos e constitui-se como alternativa aos modelos de negócio tradicionais. É levado a cabo uma sondagem junto de jornalistas e estudantes de jornalismo no Líbano, com o intuito de compreender as perspectivas dos inquiridos acerca desta alternativa de financiamento. Os resultados deste estudo exploratório revelam um nível de conhecimento reduzido no Líbano relativamente a este fenômeno, que não suscita o entusiasmo de um público concreto e é acompanhado apenas por alguns órgãos de comunicação muito concretos.

Palavras-chave: Financiamento, meios de comunicação, media, jornalismo, crowdfunding, Líbano. 


\section{Introduction}

Dans un contexte de marasme économique mondial, les modèles de financement classiques telles les banques privées ou les instances publiques perdent de leur succès surtout avec des projets en phase d'amorçage. Trouver de nouveaux moyens de ressources financières aux divers projets, nouveaux ou déjà existants, devient de nos jours un champ d'action expansif, innové, créatif et très efficace, qui dépasse les modèles classiques de financement, notamment les offres du secteur bancaire, truffées de garanties de prudences ou quasi impossibles sans caution.

L'un de ces nouveaux moyens de circuits plus courts, s'avère en croissance rapide, surtout en Europe et en Amérique, à savoir les plateformes de financement participatif (Le crowdfunding), autrement dit le financement (fondation) de la foule. Comment peut-on définir ces plateformes de financement participatif? Quels seraient leurs avantages et leurs limites?

S’appuyant sur les résultats d'une enquête en ligne s'inspirant des travaux de SANCHEZ-GONZALEZ, et PALOMO-TORRES (2014), cet article explore les connaissances des acteurs directement concernés par la restructuration des modèles de financement des médias : les journalistes et les étudiants en journalisme. Cette approche permet de vérifier jusquà quel point un public avisé est conscient des changements dans le paysage médiatique, est-il prêt à détenir le pouvoir de décision et quelles seraient ses contributions financières en termes de produits médiatiques. Le questionnaire permet également de vérifier si des campagnes de sensibilisation ou des sessions de formation sont nécessaires pour populariser la culture de crowdfunding.

\section{Revue de littérature}

\section{Définitions}

Plusieurs définitions du financement participatif ont été proposées : "Il consiste pour un porteur de projet (quel que soit son statut particulier, organisation marchande ou non marchande, etc.) à avoir recours aux services d'une plateforme de financement (généraliste ou spécialisée) afin de proposer un projet (finalisé ou non) auprès d'une communauté (large ou ciblée) de contributeurs qualifiés de soutiens (backers) en échange éventuellement de contreparties préalablement définies " (ONNEE et RENAULT, 2013, p. 54-65). 
Il s'agit du phénomène en vertu duquel un certain nombre de particuliers (la foule) peuvent, en participant chacun pour un petit montant, réunir une somme conséquente afin de financer un projet. (BERTRAND et JAKUBOWSKI, 2016, p. 38-43).

Pourtant ces définitions restent très vagues et nélucident pas l'esprit garant de son succès. En effet, le financement participatif dépasse désormais ce qu'on liait à l'offre et la demande, se dotant d'une confiance entre les deux pôles d'action : l'association, l'entreprise, la ou les personnes, porteurs d'un projet, d'une part, et des individus prêts à financer, d'autre part, dans le but principal d'assurer la liquidité nécessaire pour toute réalisation ou continuation d'un dit projet.

L'importance de ce nouveau système serait la culture du partage, de proximité, et de solidarité ce qui favorise l'esprit d'initiative pour l'autofinancement, et la synergie entre le porteur du projet et ses adhérents, connus personnellement, virtuellement, ou même inconnus. Il montre l'importance de ce qu'on pourrait considérer une intelligence collective.

Plusieurs médias internationaux se sont lancés dans l'expérience du crowdfunding avec des performances plus ou moins réussies à l'instar des sites tels que Les Jours (lancés en 2015 par d’anciens journalistes de Libération, justement pour s'affranchir des contraintes idéologiques du journal), The Correspondant ${ }^{3}$ en 2013, Krautreporter ${ }^{4}$ en 2014 and El Español en 2015. Le graphique 1 montre le gain d'intérêt de cette forme de financement sur la plateforme Kickstarter ${ }^{6}$.

Graphique 1: Nombre de journaux financés par crowdfunding selon PEW Research Center (2015)

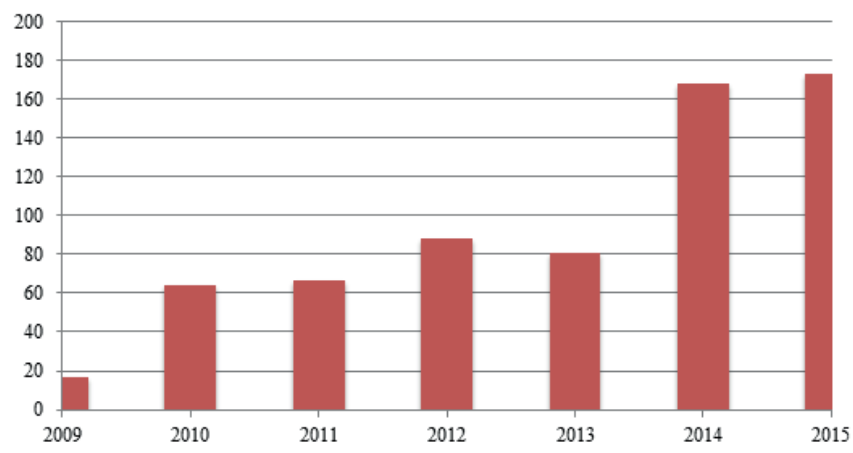

3 thecorrespondent.com

4 krautreporter.de

5 elespanol.com

6 kickstarter.com 
BELLEFLAMME et al. (2014) compare deux modèles de financement participatif. Dans le premier, les contributeurs précommandent le produit ce qui donne la possibilité aux producteurs de pratiquer une discrimination des prix entre des contributeurs plus ou moins engagés. Dans le second, les contributeurs achètent des parts qui leur permettent de partager les profits éventuels. Par une comparaison des motivations derrière les initiatives de financement participatif à travers le monde, CARVAJAL et al. (2012) montre que les projets à but non lucratif occupent plus de place en mettant en avant des principes tels que la transparence, l'engagement personnel et le contrôle sur le matériel produit.

\section{Les avantages du financement participatif}

Un des atouts majeurs du financement participatif serait l'interaction entre l'initiateur du projet et les internautes. Les porteurs de projets se voient souvrir vers de nouvelles opportunités financières, hormis le système traditionnel d'investissement et de distribution.

D’abord, le crowdfunding se développe dans une logique de partage de savoir et de décision. Le vote ainsi que la participation directe du public à un projet peuvent être sollicités au cours de la phase pré-lancement. En ce sens, le financeur contribue à définir la stratégie de l'organisation du projet. A son tour, le créateur du projet peut tester le terrain et voir si le dessein trouve un écho positif auprès du public. Il pourrait ainsi modifier ou changer le plan selon l'intérêt et la réceptivité des idées. Une fois l'objectif financier est déterminé, la collecte de fonds prend place en tenant compte de la vigueur et du dynamisme de la communauté vis-à-vis du projet.

Ensuite, la collecte de fonds auprès des internautes s'assimile à un processus d'accumulation qui peut être décomposé en trois étapes (AGRAWAL et al., 2010). Chaque phase fait intervenir des catégories différentes de contributeurs. Le créateur initie tout d'abord son capital social en l'occurrence sa famille, ses amis, et ses admirateurs. Le patrimoine relationnel de ce premier cercle, s'il est partant pour le projet, forme la deuxième catégorie de contributeurs. Vient ensuite, le troisième groupe de personnes intéressées par l'idée mais méconnues par l'initiateur du projet.

Les plateformes permettent également de s'extraire du circuit de distribution habituel en offrant aux créateurs la possibilité de mettre en vente leurs créations en ligne. Cette formule est triplement intéressante. Primo, elle permet d'accéder à un circuit de distribution, ce qui n'est pas toujours 
accessible. Secundo, elle répond à la volonté de certains créateurs de ne pas fléchir face aux directives des circuits classiques. Tertio, elle court-circuite les pourcentages de gain alloués aux entreprises et au créateur d'avoir une marge confortable, ce qui s'avère plus rentable pécuniairement.

In fine, le porteur de projet gagne en crédibilité auprès de financiers classiques, une fois que son projet attire maints investisseurs sur le net. Le succès du projet via le financement participatif lui garantit plus de notoriété et pourra récolter le financement dans la phase d'exécution du projet. Le cas échéant, l'initiateur du projet risque de perdre de crédibilité et de sa notoriété auprès des investisseurs classiques et ceux de la toile (ONNEE et RENAULT, 2013).

Plusieurs autres critères peuvent garantir la réussite des projets de crowdfunding comme l'étendu du réseau social d'amis sur Facebook (HEKMAN et BRUSSEE, 2013), le nombre élevé de «likes» (MOISSEYEV, 2013), la concentration géographique (MOLLICK, 2013) ainsi que l'étendu dans le temps (KUPPUSWAMY et BAYUS, 2015).

\section{Les désavantages du financement participatif}

Si le crowdfunding est un phénomène en pleine expansion, il demeure un modèle de financement précaire qui est soumis à plusieurs désavantages entravant son essor. Quatre inconvénients majeurs endiguent le bon fonctionnement de ce système : son récent essor, sa faible diffusion, la méfiance des investisseurs averses au risque et l'appréhension du public surtout âgé (DE BUYSERE et al, 2012).

Le deuxième bémol serait l'aléa de l'escroquerie. Les investisseurs craignent que l'argent payé ne soit pas utilisé à bon escient surtout en absence de contrat juridique. La troisième réserve est en rapport avec le temps déployé pour monter le projet en ligne, agir et réagir face aux demandes des internautes et exécuter un plan de communication professionnelle. Finalement, le risque de plagiat est fort élevé, il faut protéger les droits des initiateurs avant lélaboration et la mise en ligne du projet.

Les plateformes ne sont pas monnaie courante auprès du grand public. Le financement participatif est récent et les initiateurs de projets ne maîtrisent pas les rouages de ce système. De leur côté, les investisseurs ne sont pas tous avisés ou ignorent le fonctionnement du payement et donc sont mis hors de la sphère des promoteurs de projets. S’ajoute à cela, la méfiance des gens à payer en ligne. Ainsi, il serait capital de former voire éduquer les 
cibles potentielles pour garantir la réussite de ce mode d'investissement (DE BUYSERE et al., 2012).

Les investisseurs doivent se fier uniquement aux informations diffusées par l'initiateur du projet via la toile. En cas de fraude, non seulement la notoriété du créateur est ruinée mais aussi celle de la plateforme qui lui sert de vitrine. En 2013, par exemple, un faux projet, nommé Kobe Red, a failli récolter les 120000 \$ confiés par 3252 donateurs, fraude qui a été détectée par un groupe de journalistes enquêtant sur la plateforme Kickstarter. Dès lors, la prévention et la détection des fraudes sont d'une importance capitale pour le maintien, l’intégrité et le développement de l'industrie du financement participatif. Deux principaux leviers permettent de détecter et de déjouer la fraude:

Les réseaux sociaux peuvent servir d'une plateforme de recherche sur le profil des entrepreneurs et sur le sérieux de leur proposition. Ainsi, les investisseurs devraient bien enquêter avant de s'aventurer dans un éventuel soutien financier.

Les outils de détection de fraude qui devraient être adoptés par les plateformes pour garantir une transparence professionnelle face à un marché de plus en plus compétitif.

\section{Le financement des journaux}

La presse écrite agonise un peu partout dans le monde. Plusieurs journaux touchés par une crise sont amenés à se limiter à des parutions partielles en ligne et à licencier leurs journalistes chevronnés. Le rendement du prix de vente ou d'abonnement, et les recettes publicitaires ne suffisent plus à couvrir les frais d'impression et les salaires des employés. La presse écrite voit son existence en danger.

La France, par exemple, a vu disparaitre deux journaux en 2012, FranceSoir et La Tribune. Le quotidien Libération, qui y a échappé de justesse, a licencié le tiers de ses employés en septembre 2014. Durant cette même année, le rachat du Nouvel Observateur par la société «Le Monde Libre »a exigé la clause de cession de près de 15\% de la rédaction. Quant au Figaro, il accroit les plans de « départs volontaires ». En Espagne, près de 200 médias ont cessé de paraitre entre 2008 et 2012 et en Allemagne, plus de 1000 emplois ont été rompus en 2013 (CAGE, 2015). 
Aux Etats-Unis le secteur n'est guère meilleur. Le site Newspaper Death Watch $^{7}$ fait le deuil de 12 quotidiens locaux disparus depuis 2007 et en compte autant " en voie de disparition ", jusqu'au point où de nombreux comtés nont plus aucun journal. Le Chicago Tribune et le Los Angeles Times ont tous deux été mis en faillite en 2008, année durant laquelle les journaux américains ont affiché 15000 pertes d'emplois (SCHIFFRIN, 2010).

Cette crise est loin de toucher uniquement les pays européens et américains. Le Moyen-Orient vit la même secousse. Jusqu'ici, les journaux libanais sont appuyés par un nombre réduit d’actionnaires, ou par des dons remarquables offerts par des mercenaires. Le modèle de financement d'une foule nest pas encore connu dans ce secteur.

Les réformes dans ce secteur demeurent indispensables car les sources de financement majeures sont limitées à la publicité. Depuis l'arrivée d'Internet, qui lui offre un espace infini, deux phénomènes ont lieu en parallèle: les prix de la publicité en ligne sont tirés vers le bas, alors que l'intégralité des revenus est captée par une poignée d'acteurs dominants, dont Facebook et Google.

L'introduction de "murs payants" est la seule solution viable sur le long terme pour les médias d'information politique et générale (CAGE, 2015). Une formule déjà adoptée par plusieurs médias au Liban, dont L'Orient-Le Jour, le seul journal francophone au pays. En outre, la presse libanaise est fragilisée par sa dépendance historique vis-à-vis d'États étrangers et surtout arabes. Par exemple, la baisse des revenus du pétrole engendre une diminution des financements des journaux libanais, et par conséquent du nombre de journalistes, ainsi que de l'originalité et de la qualité du contenu produit par les médias. Autre conséquence de la baisse des revenus : le rachat de journaux par des hommes d'affaires (à l'instar du modèle de rachat du Washington Post en 2013 par Amazon), ce qui risque de créer les risques de conflit d'intérêts pour les rédactions. Cette très forte concentration actionnariale met également le journal à la merci de la santé financière de son propriétaire.

Le modèle actionnarial traditionnel n'est au demeurant pas le plus adapté au fonctionnement des médias car la recherche de rentabilité à court terme enfermerait le journal dans un cercle vicieux de baisse de la qualité et du lectorat (CAGE, 2015). Autre piste : le modèle des fondations - à but non lucratif - qui permettent au capital investi dêtre pérennisé, le donateur ne pouvant pas retirer son apport à tout moment. Mais ce modèle concentre

7 newspaperdeathwatch.com/ 
trop de pouvoir dans les mains du conseil d'administration, souvent dominé par une même famille, critique l'économiste. Tel est le cas de la fondation allemande Bertelsmann, propriétaire du groupe Bertelsmann (RTL Group, Prisma Media...). Quant au financement participatif pur, il ne permettrait pas de réunir suffisamment de fonds pour lancer un média, même de petite taille.

Un modèle « hybride entre une fondation et une société par action » risque démerger : la société de média à but non lucratif. Dans ce modèle, des petits donateurs - comme des journalistes ou des lecteurs - peuvent devenir actionnaires, avec un droit de vote plus important que leur apport en capital.

Au-delà de la levée de fonds, les créateurs de projets de crowdfunding sont à la recherche d'une validation publique du bien-fondé de leurs idées, de la création d'un réseau et d'une diffusion sociale de leurs idées. Au-delà de la recherche de profits, les contributeurs visent à contribuer financièrement à la réussite d'un projet et à participer activement à une communauté de création digne de confiance (GERBER et al., 2012). Le financement participatif solidifie le lien entre les lecteurs et les journalistes, ce qui pousse ces derniers à remettre en cause leur rôle et de réaffirmer leur mission de responsabilité vis-à-vis des lecteurs (AITAMURTO, 2011).

Pour les lecteurs, l'objectif est plus altruiste que matérialiste : ils ont l'impression qu'ils prennent part à la production du bien commun en finançant le projet journalistique, surtout lorsqu’il s'agit de bénéfices pour la communauté (BELLEFLAMME et al., 2014). Ils veulent prendre part au processus de création et de production et non seulement recevoir le produit fini. En retour, les journalistes doivent bien commercialiser leur projet en ciblant un objectif précis permettant de générer suffisamment d’enthousiasme de la part du public (AITAMURTO, 2011).

En attendant, pour les médias déjà installés depuis des décennies au Liban, reste aussi la solution d'un apport de fonds publics pour aider le secteur à surmonter ses difficultés. Pour autant, l'économiste ne plaide pas pour une transposition du système de subventions similaire à celui en vigueur en France.

Le nouveau secteur de financement parait un moyen efficace pour assurer la continuation de parution de ces journaux, surtout que c'est un projet en cours qui pourrait englober des participants de dons gratuits, avec des montants minimes ou raisonnables, comme il pourrait attirer les aspirateurs aux profits, en leur impliquant à une synergie et coopération interac- 
tive pour mieux soutenir le journal menacé, surtout par l'élargissement des abonnés et des intéressés aux publicités offertes, sans exclure la contribution gratuite des articles offerts par des nouveaux auteurs et écrivains.

Ce système assure une certaine aide, moyennant une interdépendance à favoriser entre ces deux parties pôles, dans le respect que chacun prête selon son propre rôle et position, sans arriver à remplacer l'auteur du projet ou à compromettre son action, ni à caser les participants dans la passivité des simples donateurs bienfaiteurs.

Si les anciennes générations de journalistes préfèrent le système classique, et séloignent de toute sorte de gestion moderne, l'idée commence à séduire la nouvelle génération d'étudiants en journalisme, enthousiastes et très à l'aise avec les nouvelles technologies. L’enquête menée dans le cadre de cet article permet de diagnostiquer la situation au Liban.

\section{Méthodologie}

Larticle adopte une approche quantitative pour sonder les opinions d'une population de journalistes déjà engagés dans une carrière professionnelle et des étudiants dans un programme formel de formation aux métiers de journalisme. Lobjectif de cette étude est de savoir si le crowdfunding peut constituer une alternative viable aux modes traditionnels de financement des médias (abonnements, publicités, mécénat, subventions publiques, financement politique...). Si cette alternative est suffisamment ancrée dans l'esprit et dans les pratiques des journalistes actuels et des futurs journalistes, elle permettra de créer un nouveau modèle économique pour les médias. Comme ce mode de financement n'est pas encore très utilisé, l'étude se limiterait à une approche exploratoire.

Pour sonder les opinions de cette population a priori spécialisée dans le domaine journalistique, une enquête par Internet (à travers la plateforme de Google Forms) a été menée auprès d'un échantillon de 65 répondants dont les caractéristiques sociodémographiques sont résumées dans le tableau ci-dessous. Le choix de léchantillon a été fait selon la méthode d'échantillonnage par jugement car les répondants doivent avoir une expérience ou une initiation au sujet et directement concernés par la problématique. Le critère retenu est la profession : les journalistes qui exercent déjà ce métier ou bien les journalistes en herbe pour détecter combien le phénomène de crowdfunding est diffusé. Les étudiants sont pour la grande majorité des étudiants en journalisme (toutes spécialisations confondues) de l'Université 
Antonine et de l'Université Libanaise, sans égard pour la religion, la région, la classe sociale... Le taux de réponse obtenu au questionnaire est de 72\% sur une durée de 15 jours (mai-juin 2017).

Léchantillon est constitué principalement de jeunes femmes, étudiantes en journalisme, ayant une expérience relativement récente et choisissant la télé comme domaine de prédilection pour leur carrière. Loin de prétendre être exhaustif, cet échantillon permet de donner une vue d’ensemble de la population active dans le secteur du journalisme au Liban. Le simple fait que l'enquête a été menée par Internet prédéfinit le profil des répondants.

Tableau 1: Statistiques descriptives de l'échantillon

\begin{tabular}{|l|l|}
\hline Nombre de répondants & 65 \\
\hline Pourcentage de femmes & $53 \%$ \\
\hline Tranche d'âge 21-25 & $63.10 \%$ \\
\hline Pourcentage d'étudiants en journalisme & $52.30 \%$ \\
\hline TV: domaine de prédilection & $31.30 \%$ \\
\hline $\begin{array}{l}\text { Expérience de moins de 5 ans } \\
\text { (pour les journalistes professionnels) }\end{array}$ & $51.60 \%$ \\
\hline
\end{tabular}

Le questionnaire se divise principalement en 3 grandes parties. D’abord, les questions visent à vérifier si le concept de financement participatif est familier au répondant, si les plateformes de financement lui sont connues et s'il a pris part de près ou de loin à de tels projets. Ensuite, le questionnaire tente de cerner les représentations des répondants concernant le crowdfunding à travers leurs opinions sur les possibilités ouvertes par le financement participatif, son impact sur le secteur du journalisme, les règles à respecter et les contraintes à placer à ce mode de financement alternatif. Enfin, des questions interrogent les répondants sur leur expérience personnelle dans le passé et éventuellement dans le futur en ce qui concerne le choix des projets à financer, le montant des contributions et les projets qui en bénéficieraient. 


\section{Analyse des résultats}

L'enquête sert d'une étude exploratoire des perceptions des actants du domaine du journalisme concernant le crowdfunding. L'analyse des résultats n'a pas l'intention dêtre concluante ou de servir de grille de lecture exhaustive. Léchantillon choisi n'est pas significativement de taille pour que les résultats obtenus soient généralisables. D’ailleurs, les tests de signification échouent souvent à tester la validité des croisements entre les variables ou bien la comparaison entre les moyennes. De ce fait, les interprétations qui suivent devraient être vérifiées par un travail empirique plus extensif. Elles permettent, tout de même, à ouvrir des pistes d'interrogations qui touchent essentiellement lavenir du crowdfunding comme mode viable de financement des projets journalistiques.

Lintitulé des questions relatives à certaines variables sociodémographiques est résumé dans le tableau ci-dessous afin de mieux lire le croisement des opinions avec le profil des répondants. Chaque variable correspond à une question posée au cours de l'enquête.

Tableau 2: Libellé des variables sociodémographiques

\begin{tabular}{|c|l|}
\hline Variable & \multicolumn{1}{c|}{ Intitulé de la question } \\
\hline Etudiant & $\begin{array}{l}\text { Etes-vous actuellement inscrit dans un } \\
\text { programme de formation au journalisme? }\end{array}$ \\
\hline Domaine & $\begin{array}{l}\text { Dans quel domaine médiatique avez-vous } \\
\text { l'intention de poursuivre votre carrière? }\end{array}$ \\
\hline Expérience & $\begin{array}{l}\text { Combien d'années d'expérience } \\
\text { journalistique avez-vous? }\end{array}$ \\
\hline Familier & $\begin{array}{l}\text { Etes-vous familier avec le phénomène } \\
\text { de crowdfunding? }\end{array}$ \\
\hline
\end{tabular}

\section{Familiarité}

Le croisement entre les variables sociodémographiques et la variable Familier donne les résultats résumés dans le tableau ci-dessous: 
Tableau 3: Croisement de la Familiarité par variables de profil

\begin{tabular}{|c|c|c|c|c|}
\hline & & \multicolumn{3}{|c|}{ Familier } \\
\hline & & Non & Oui & Pas sûr \\
\hline \multirow{5}{*}{ Age } & [21-25] & $25.6 \%$ & $38.5 \%$ & $35.9 \%$ \\
\hline & [26-30] & - & $66.7 \%$ & $33.3 \%$ \\
\hline & [31-40] & $22.2 \%$ & $55.6 \%$ & $22.2 \%$ \\
\hline & {$[41-50]$} & - & - & $100.0 \%$ \\
\hline & Plus de 50 & $100.0 \%$ & - & - \\
\hline \multirow[t]{2}{*}{ Sexe } & Masculin & $25.0 \%$ & $16.7 \%$ & $58.3 \%$ \\
\hline & Féminin & $20.8 \%$ & $50.0 \%$ & $29.2 \%$ \\
\hline \multirow[t]{2}{*}{ Etudiant } & Non & $17.2 \%$ & $41.4 \%$ & $41.4 \%$ \\
\hline & Oui & $25.8 \%$ & $45.2 \%$ & $29.0 \%$ \\
\hline \multirow[t]{5}{*}{ Domaine } & TV & $44.4 \%$ & $27.8 \%$ & $27.8 \%$ \\
\hline & Radio & - & $50.0 \%$ & $50.0 \%$ \\
\hline & $\begin{array}{c}\text { Journaux } \\
\text { et magazines }\end{array}$ & - & $50.0 \%$ & $50.0 \%$ \\
\hline & Web Journalisme & - & $100.0 \%$ & - \\
\hline & $\begin{array}{l}\text { Journalisme } \\
\text { Free Lance }\end{array}$ & - & $60.0 \%$ & $40.0 \%$ \\
\hline \multirow[t]{3}{*}{ Expérience } & Moins que 5 ans & $12.5 \%$ & $43.8 \%$ & $43.8 \%$ \\
\hline & Entre 5 et 10 ans & - & $80.0 \%$ & $20.0 \%$ \\
\hline & Plus que 10 ans & $37.5 \%$ & $12.5 \%$ & $50.0 \%$ \\
\hline
\end{tabular}


Le concept de crowdfunding est familier pour les jeunes, les répondants de sexe féminin et ayant une expérience modérée dans le domaine journalistique. Les étudiants en journalisme ne sont pas encore complètement familiarisés avec le concept (de même pour ceux qui ne sont pas actuellement en formation). La familiarité reste limitée en fonction du domaine de prédilection des répondants. Cette familiarité est surtout répandue parmi les répondants qui souhaitent poursuivre leur carrière dans le domaine du journalisme en dehors des medias audio-visuels. Cela peut être dû à une prise de conscience des difficultés financières que vit ce secteur alors que les télévisions et les radios libanaises ne souffrent pas des mêmes contraintes.

\section{Définition}

Dans une tentative de mieux cerner la connaissance du concept par les répondants, 3 définitions leur ont été proposées pour détecter celle qui leur semble la plus pertinente. Les définitions ont été posées en anglais, ce qui pourrait dérouter certains répondants. Le tableau ci-dessous en retranscrit la traduction:

Tableau 4: Propositions de définitions

\begin{tabular}{|l|l|}
\hline DEF1 & $\begin{array}{l}\text { Lactivité consistant à financer un projet ou une entreprise en collec- } \\
\text { tant des contributions monétaires d'un grand nombre de personnes, } \\
\text { généralement via Internet }\end{array}$ \\
\hline DEF2 & $\begin{array}{l}\text { Un appel au public, essentiellement via Internet, pour l'approvision- } \\
\text { nement en de ressources financières soit sous forme de donation, soit } \\
\text { en échange de droits ou de récompense et / ou de droits de vote dans } \\
\text { le but de soutenir des initiatives à des fins spécifiques }\end{array}$ \\
\hline DEF3 & $\begin{array}{l}\text { Une méthode innovante pour financer une variété de nouvelles en- } \\
\text { treprises, permettant aux fondateurs individuels de projets à but lu- } \\
\text { cratif, culturels ou sociaux de demander un financement à un grand } \\
\text { nombre d'individus }\end{array}$ \\
\hline
\end{tabular}


Tableau 5: Croisement des définitions par variables de profil

\begin{tabular}{|c|c|c|c|c|c|c|c|c|}
\hline \multirow{2}{*}{$\stackrel{0}{.}$} & \multicolumn{2}{|c|}{ Sexe } & \multicolumn{6}{|c|}{ Age } \\
\hline & Homme & Femme & [21-25] & [26-30] & [31-40] & & 50] & $\begin{array}{l}\text { Plus de } \\
50 \text { ans }\end{array}$ \\
\hline DEF1 & $15,8 \%$ & $84,2 \%$ & $63,2 \%$ & $10,5 \%$ & $21,1 \%$ & & & $5,3 \%$ \\
\hline DEF2 & $10,7 \%$ & $89,3 \%$ & $60,7 \%$ & $17,9 \%$ & $14,3 \%$ & & & - \\
\hline DEF3 & $35,3 \%$ & $64,7 \%$ & $64,7 \%$ & $17,6 \%$ & $17,6 \%$ & & & - \\
\hline \multirow{2}{*}{ : } & \multicolumn{2}{|c|}{ Etudiant } & \multicolumn{6}{|c|}{ Experience } \\
\hline & Non & Oui & $<5$ ans & Entr & 5 et $10 a$ & & $\mathrm{Plu}$ & de 10 ans \\
\hline DEF1 & $47,4 \%$ & $52,6 \%$ & $55,6 \%$ & & $11,1 \%$ & & & $33,3 \%$ \\
\hline DEF2 & $57,1 \%$ & $42,9 \%$ & $50,0 \%$ & & $25,0 \%$ & & & $25,0 \%$ \\
\hline DEF3 & $35,3 \%$ & $64,7 \%$ & $50,0 \%$ & & $16,7 \%$ & & & $33,3 \%$ \\
\hline
\end{tabular}

\begin{tabular}{|c|c|c|c|c|c|c|c|c|}
\hline & \multicolumn{5}{|c|}{ Domaine } & \multicolumn{3}{c|}{ Familier } \\
\cline { 2 - 9 } & TV & Radio & $\begin{array}{c}\text { Journaux } \\
\text { Mag. } \\
\text { Nateb }\end{array}$ & $\begin{array}{c}\text { Webrnal- } \\
\text { isme }\end{array}$ & $\begin{array}{c}\text { Jour. } \\
\text { Free- } \\
\text { Lance }\end{array}$ & Non & Oui & $\begin{array}{c}\text { Pas } \\
\text { sûr }\end{array}$ \\
\hline DEF1 & $60.0 \%$ & $10.0 \%$ & - & $20.0 \%$ & $10.0 \%$ & $36.8 \%$ & $31.6 \%$ & $31.6 \%$ \\
\hline DEF2 & $63.6 \%$ & - & $18.2 \%$ & $9.1 \%$ & $9.1 \%$ & $11.1 \%$ & $55.6 \%$ & $33.3 \%$ \\
\hline DEF3 & $54.5 \%$ & $18.2 \%$ & - & - & $27.3 \%$ & $21.4 \%$ & $35.7 \%$ & $42.9 \%$ \\
\hline
\end{tabular}


La première définition insiste sur le rôle que peut jouer Internet dans la collecte des fonds nécessaires. Cette définition suscite l'intérêt des femmes, des jeunes, des étudiants, ceux qui ont une jeune expérience dans le domaine journalistique, qui choisissent la télé comme domaine de prédilection et qui ne sont pas forcément familiarisés avec le phénomène de crowdfunding.

La deuxième définition insiste sur la contrepartie attendue des contributions volontaires. Elle suscite l'intérêt du même profil de répondants que la définition précédente.

La troisième définition met l'emphase sur la nature des projets qui peuvent être financés par crowdfunding. Elle suscite l'intérêt surtout des jeunes étudiants qui trouvent dans cette forme de financement une possibilité pour rendre leurs projets réalisables.

\section{Plateforme}

Tableau 6: Connaissance des plateformes de crowdfunding

\begin{tabular}{|l|c|c|c|c|c|}
\hline & \multicolumn{4}{|l|}{ Parmi les plateformes de crowdfunding suivantes, lesquelles: } \\
\cline { 2 - 6 } & $\begin{array}{c}\text { CROWD- } \\
\text { CUBE }\end{array}$ & $\begin{array}{c}\text { KICK- } \\
\text { STARTER }\end{array}$ & $\begin{array}{c}\text { INDIEGO- } \\
\text { GO }\end{array}$ & BEACON & ZOOMAAL \\
\hline $\begin{array}{l}\text { En avez-vous } \\
\text { entendu } \\
\text { parler? }\end{array}$ & 46,7 & 62,2 & 58,6 & 62,5 & 48,7 \\
\hline $\begin{array}{l}\text { Avez-vous } \\
\text { visité? }\end{array}$ & 30,0 & 16,2 & 17,2 & 25,0 & 28,2 \\
\hline $\begin{array}{l}\text { Etes-vous } \\
\text { familier avec? }\end{array}$ & 6,7 & 2,7 & 10,3 & 3,1 & 10,3 \\
\hline $\begin{array}{l}\text { Y avez-vous } \\
\text { contribué? }\end{array}$ & 16,7 & 18,9 & 13,8 & 9,4 & 12,8 \\
\hline Total & 100,0 & 100,0 & 100,0 & 100,0 & 100,0 \\
\hline
\end{tabular}

Les répondants ont entendu parler des plateformes les plus connues au monde, sans qu'ils n'y contribuent. Très peu de répondants ont déjà interagi avec ces plateformes. Si ces plateformes sont majoritairement connues, cela ne suffit pas pour encourager les répondants à se familiariser avec elles ou bien à effectuer des contributions à des projets financés collectivement. La 
plateforme $Z O O M A A L^{8}$ qui est le leader parmi les plateformes arabes reste relativement méconnue des répondants. Cette méconnaissance ne se justifie pas uniquement par un manque d'implication de la part des répondants dans le financement alternatif due à une désinformation ou bien une appréhension liée aux risques, mais également par un manque d'efficacité de la stratégie marketing de la plateforme.

\section{Connaissance}

Tableau 7: Croisement entre le financement des projets journalistiques et les variables de profil

\begin{tabular}{|c|c|c|c|}
\hline & \multicolumn{2}{|c|}{$\begin{array}{l}\text { Saviez-vous que les projets de journalisme peuvent } \\
\text { également être financés par crowdfunding? }\end{array}$} \\
\hline & & Non & Oui \\
\hline \multirow{5}{*}{ Age } & [21-25] & $43,9 \%$ & $56,1 \%$ \\
\hline & [26-30] & $40,0 \%$ & $60 \%$ \\
\hline & [31-40] & $54,5 \%$ & $45,5 \%$ \\
\hline & [41-50] & $100,0 \%$ & - \\
\hline & Plus de 50 & - & $100,0 \%$ \\
\hline \multirow{2}{*}{ Sexe } & Masculin & $41,7 \%$ & $58,3 \%$ \\
\hline & Féminin & $47,2 \%$ & $52,8 \%$ \\
\hline \multirow{2}{*}{ Etudiant } & Non & $51,6 \%$ & $48,4 \%$ \\
\hline & Oui & $41,2 \%$ & $58,8 \%$ \\
\hline \multirow{5}{*}{ Domaine } & TV & $45,0 \%$ & $55,0 \%$ \\
\hline & Radio & $66,7 \%$ & $33,3 \%$ \\
\hline & $\begin{array}{l}\text { Journaux et } \\
\text { magazines }\end{array}$ & $50,0 \%$ & $50,0 \%$ \\
\hline & $\begin{array}{c}\text { Web } \\
\text { Journalisme }\end{array}$ & - & $100,0 \%$ \\
\hline & $\begin{array}{l}\text { Journalisme } \\
\text { Free-lance }\end{array}$ & $20,0 \%$ & $80,0 \%$ \\
\hline
\end{tabular}




\begin{tabular}{|c|c|c|c|}
\hline Expérience & $\begin{array}{c}\text { Moins que } \\
5 \text { ans }\end{array}$ & $56,3 \%$ & $43,8 \%$ \\
\cline { 2 - 4 } & $\begin{array}{c}\text { Entre } \\
5 \text { et } 10 \text { ans }\end{array}$ & $50,0 \%$ & $50,0 \%$ \\
\cline { 2 - 4 } & $\begin{array}{c}\text { Plus que } \\
10 \text { ans }\end{array}$ & $44,4 \%$ & $55,6 \%$ \\
\hline \multirow{2}{*}{ Familier } & Non & $61,5 \%$ & $38,5 \%$ \\
\cline { 2 - 4 } & Oui & $30,8 \%$ & $69,2 \%$ \\
\cline { 2 - 4 } & Pas sûr & $52,4 \%$ & $47,6 \%$ \\
\hline
\end{tabular}

La possibilité de financer des projets de journalisme reste relativement méconnue pour les répondants. Les jeunes (âgés entre 21 et 30 ans) semblent y être sensibilisés ainsi que les étudiants, ceux qui travaillent dans le domaine du journalisme indépendant, ceux qui ont de l'expérience et ceux qui sont familiarisés avec le concept de crowdfunding. Cette question montre que le crowdfunding aura un avenir avec les jeunes générations. Les jeunes sont conscients de la possibilité de profiter de ces alternatives de financement surtout s'ils envisagent de faire carrière dans des medias décentralisés tel que le webjournalisme. L'influence de l'expérience sur cette prise de conscience reste mitigée. Il est étonnant de voir que les réponses sont symétriques en fonction des années d'expérience. Les moins expérimentés ne sont pas encore suffisamment immergés dans les problèmes de financement pour qu'ils se fassent une idée claire des alternatives. Les plus expérimentés sont davantage conscients des insuffisances des moyens de financement et sont plus ouverts aux alternatives.

\section{Représentations}

Afin d'identifier les représentations du crowdfunding chez les répondants une série d'affirmations leur a été proposée sur laquelle ils expriment leur accord ou non. Encore une fois, les propositions sont formulées en anglais et le tableau ci-dessous en présente la traduction. 
Tableau 8: Représentations possibles du crowdfunding

\begin{tabular}{|c|c|}
\hline Code & Affirmation \\
\hline CROWD1 & $\begin{array}{l}\text { Le crowdfunding représente une opportunité pour la carrière des } \\
\text { étudiants en journalisme }\end{array}$ \\
\hline CROWD2 & $\begin{array}{l}\text { Le crowdfunding est une opportunité pour les journalistes touchés } \\
\text { par la fermeture des médias ou par les licenciements collectifs }\end{array}$ \\
\hline CROWD3 & $\begin{array}{l}\text { Le crowdfunding facilite le lancement des projets mais ne garantit pas } \\
\text { leur soutenabilité financière }\end{array}$ \\
\hline CROWD4 & $\begin{array}{l}\text { Le crowdfunding soutient l'émergence de médias spécialisés qui } \\
\text { ciblent une audience précise }\end{array}$ \\
\hline CROWD5 & $\begin{array}{l}\text { Le crowdfunding facilite la création de projets médiatiques de } \\
\text { manière plus indépendante financièrement que les modes tradition- } \\
\text { nels de financement }\end{array}$ \\
\hline CROWD6 & $\begin{array}{l}\text { Le crowdfunding ouvre la voie aux journalistes d'une forme de jour- } \\
\text { nalisme plus libérée }\end{array}$ \\
\hline CROWD7 & $\begin{array}{l}\text { Certains projets médiatiques innovateurs n'auraient pas pu voir le jour } \\
\text { sans le crowdfunding }\end{array}$ \\
\hline CROWD8 & $\begin{array}{l}\text { Le crowdfunding garantit que seuls les produits ayant une forte de- } \\
\text { mande trouvent un financement }\end{array}$ \\
\hline CROWD9 & $\begin{array}{l}\text { Crowdfunding est une opportunité pour développer un journalisme } \\
\text { plus participatif plus proche du public }\end{array}$ \\
\hline CROWD10 & $\begin{array}{l}\text { Crowdfunding change le concept du journalisme comme une activité } \\
\text { d'intérêt général }\end{array}$ \\
\hline CROWD11 & $\begin{array}{l}\text { Les initiateurs de projets financés par crowdfunding sont tenus res- } \\
\text { ponsables devant leur public }\end{array}$ \\
\hline
\end{tabular}

Pour chacune des affirmations, le répondant à la possibilité de répondre sur une échelle de préférence allant de 1 : Pas du tout d'accord à 5: Tout à fait d'accord. Pour chacune des représentations, les réponses ont été comptabilisées en pourcentage. La dernière colonne représente la moyenne de ces pourcentages pour les différentes affirmations. 
Tableau 9: Pourcentage des réponses par représentation

\begin{tabular}{|l|c|c|c|c|c|c|}
\hline & CROWD1 & CROWD2 & CROWD3 & CROWD4 & CROWD5 & CROWD6 \\
\hline $\begin{array}{l}\text { Pas du tout } \\
\text { d'accord }\end{array}$ & 3,5 & 3,7 & 1,8 & 5,6 & 2,0 & 1,9 \\
\hline Pas d'accord & 7,0 & 3,7 & 7,0 & 11,1 & 5,9 & 13,0 \\
\hline Neutre & 19,3 & 33,3 & 28,1 & 31,5 & 27,5 & 22,2 \\
\hline D'accord & 50,9 & 38,9 & 38,6 & 44,4 & 47,1 & 44,4 \\
\hline $\begin{array}{l}\text { Tout à fait } \\
\text { d'accord }\end{array}$ & 19,3 & 20,4 & 24,6 & 7,4 & 17,6 & 18,5 \\
\hline Total & 100,0 & 100,0 & 100,0 & 100,0 & 100,0 & 100,0 \\
\hline
\end{tabular}

\begin{tabular}{|l|c|c|c|c|c|c|}
\hline & CROWD7 & CROWD8 & CROWD9 & CROWD10 & CROWD11 & Moyenne \\
\hline $\begin{array}{l}\text { Pas du tout } \\
\text { d'accord }\end{array}$ & 7,4 & 9,3 & - & 9,4 & 5,6 & $\mathbf{5 , 0}$ \\
\hline Pas d'accord & 7,4 & 16,7 & 9,3 & 18,9 & 13,0 & $\mathbf{1 0 , 3}$ \\
\hline Neutre & 33,3 & 33,3 & 38,9 & 32,1 & 42,6 & $\mathbf{3 1 , 1}$ \\
\hline D'accord & 37,0 & 31,5 & 37,0 & 26,4 & 29,6 & $\mathbf{3 8 , 7}$ \\
\hline $\begin{array}{l}\text { Tout à fait } \\
\text { d'accord }\end{array}$ & 14,8 & 9,3 & 14,8 & 13,2 & 9,3 & $\mathbf{1 5 , 4}$ \\
\hline Total & 100,0 & 100,0 & 100,0 & 100,0 & 100,0 & $\mathbf{1 0 0 , 0}$ \\
\hline
\end{tabular}

Lattitude générale de la population enquêtée reste mitigée. En moyenne, les affirmations proposées suscitent une attitude neutre (pour 31.1\%) ou favorable (pour 38.7\%). Les caractéristiques du crowdfunding ne génèrent ni rejet catégorique (5\%) ni une acceptation enthousiaste (15.4\%).

Les représentations $1,3,5$ et 6 , génèrent le plus d’enthousiasme car le pourcentage de répondants ayant donné des réponses « d'accord » et « tout à fait d'accord » dépasse les $60 \%$ et atteint $70 \%$ pour la première affirmation. Le point commun entre ces affirmations est le mot " possibilité ». Elles restent très générales dans leur appréciation du phénomène et de ses conséquences matérielles ou financières.

\section{Contribution}

Afin dévaluer le degré d'engagement dans le processus de financement alternatif des projets journalistiques et la viabilité financière de ce mode de 
financement, il a été demandé aux répondants de déclarer quel est le montant maximal qu'ils étaient prêts à payer comme contribution à un projet de crowdfunding et pour quel genre de projet ils seraient le plus sensibles.

Tableau 10: Projets à financier par crowdfunding

\begin{tabular}{|l|c|c|}
\hline \multicolumn{2}{|c|}{$\begin{array}{c}\text { PROJET “Lequel des projets journalistiques suivants seriez-vous } \\
\text { le plus enthousiaste à financer?" }\end{array}$} \\
\hline Projet & Fréquence & Pourcentage \\
\hline Application & 4 & 6.2 \\
\hline Blog & 1 & 1.5 \\
\hline Livre & 4 & 6.2 \\
\hline Documentaire & 12 & 18.5 \\
\hline Magazine & 6 & 9.2 \\
\hline Journal & 5 & 7.7 \\
\hline Sans format spécifique & 7 & 10.8 \\
\hline Photojournalisme & 2 & 3.1 \\
\hline Radio/Podcast & 9 & 13.8 \\
\hline Website & 15 & 23.1 \\
\hline Total & $\mathbf{6 5}$ & $\mathbf{1 0 0}$ \\
\hline
\end{tabular}

À la question concernant le type de projet journalistique, les répondants étaient le plus enthousiasmés par le financement de websites $(23.1 \%)$ et de documentaires (18.5\%). Ces deux réponses peuvent être justifiées par la nécessité de financer des projets d'utilité publique. L'intérêt à financer des journaux est très faible (7.7\%), ce qui marque un désintérêt des formes traditionnelles de journalisme. Ce refus pose un problème majeur concernant l'avenir des journaux qui souffrent de l'insuffisance des moyens traditionnels de générer des fonds et qui ne suscitent pas apparemment l'intérêt même des principaux concernés par le phénomène. Ce manque d'intérêt, s’il est généralisé, marquera une déviation dans les formats qu'emprunte l'information journalistique, mais pas dans le contenu, puisque le financement de website est le projet qui suscite le plus d'intérêt.

Ces deux formes de projets ont un modèle économique particulier. Le financement de documentaire ne constitue pas une ligne de financement permanent, mais un financement ponctuel, suffisamment encadré. Le con- 
tributeur bénéficierait d'une visibilité à sa contribution. D’autre part, le financement de websites journalistiques appelle à un éclatement du mode de production, vers un modèle économique moins onéreux qui associerait crowdfunding et crowdsourcing (littéralement « approvisionnement par la foule ", lorsqu'un media fait appel au public pour lui fournir un contenu publiable) afin d'alléger les coûts de production.

Tableau 11: Contribution individuelle aux projets de journalisme

\begin{tabular}{|l|c|c|}
\hline \multicolumn{3}{|c|}{$\begin{array}{c}\text { CONTRIBUTION “Combien êtes-vous prêts à contribuer pour financier un } \\
\text { projet de journalisme financé par crowdfunding?” }\end{array}$} \\
\hline & Fréquence & Pourcentage \\
\hline 0 USD & 4 & 6.2 \\
\hline$[\mathbf{0 - 2 5}$ USD] & 9 & 13.8 \\
\hline$[\mathbf{2 6 - 1 0 0}$ USD] & 25 & 38.5 \\
\hline$[\mathbf{1 0 1 - 2 5 0}$ USD] & 11 & 16.9 \\
\hline$[\mathbf{2 5 1 - 5 0 0 ~ U S D ]}$ & 7 & 10.8 \\
\hline$[\mathbf{5 0 1 - 1 0 0 0 ~ U S D ]}$ & 5 & 7.7 \\
\hline Plus de 1000 USD & 4 & 6.2 \\
\hline Total & 65 & 100.0 \\
\hline
\end{tabular}

A cet effet, $38.5 \%$ des répondants sont prêts à payer un montant compris entre 26 et 100 USD pour financer un projet journalistique. Aux deux extrêmes de léchelle des contributions, le pourcentage est le plus faible, ce qui montre que les répondants sont conscients que les petites contributions sont insuffisantes, mais en même temps, ils ne sont pas suffisamment impliqués par ce mode de financement pour proposer des sommes conséquentes. Cette contribution entre 26 et 100 USD ne peut à elle seule suffire pour subvenir aux besoins financiers d'un media, et surtout d'un journal. Si cette tendance se concrétise, le crowdfunding ne pourrait pas remplacer les modes de financement traditionnels. Il peut servir de complément de financement, à moins qu'il y ait une restructuration dans les medias eux-mêmes, vers un modèle économique moins coûteux.

D'autre part, cette contribution modeste est conforme aux projets médiatiques que les répondants ont eu l'intention de financer (documentaire et websites). La tendance serait à un allègement à la fois des coûts et des contributions. 
Tableau 12: Ventilation des contribution par variables de profil

\begin{tabular}{|l|c|c|c|c|c|c|c|}
\hline \multirow{2}{*}{$\begin{array}{l}\text { Contribu- } \\
\text { tion }\end{array}$} & \multicolumn{2}{|c|}{ Sexe } & \multicolumn{3}{c|}{ Age } & \multicolumn{2}{c|}{ Etudiant } \\
\cline { 2 - 8 } & Masculin & Féminin & {$[\mathbf{2 1 - 2 5}]$} & {$[\mathbf{2 6 - 3 0 ]}$} & {$[31-40]$} & Non & Oui \\
\hline $\mathbf{0}$ USD & - & $100,0 \%$ & $75,0 \%$ & $25,0 \%$ & - & $75,0 \%$ & $25,0 \%$ \\
\hline $\begin{array}{l}\text { [0-25 } \\
\text { USD] }\end{array}$ & $22,2 \%$ & $77,8 \%$ & $77,8 \%$ & $11,1 \%$ & $11,1 \%$ & $33,3 \%$ & $66,7 \%$ \\
\hline $\begin{array}{l}\text { [26-100 } \\
\text { USD] }\end{array}$ & $20,0 \%$ & $80,0 \%$ & $60,0 \%$ & $16,0 \%$ & $16,0 \%$ & $60,0 \%$ & $40,0 \%$ \\
\hline $\begin{array}{l}\text { [101-250 } \\
\text { USD] }\end{array}$ & $27,3 \%$ & $72,7 \%$ & $45,5 \%$ & $9,1 \%$ & $45,5 \%$ & $63,6 \%$ & $36,4 \%$ \\
\hline $\begin{array}{l}\text { [251-500 } \\
\text { USD] }\end{array}$ & - & $100,0 \%$ & $85,7 \%$ & - & $14,3 \%$ & $14,3 \%$ & $85,7 \%$ \\
\hline $\begin{array}{l}\text { [501-1000 } \\
\text { USD] }\end{array}$ & - & $100,0 \%$ & $60,0 \%$ & $20,0 \%$ & - & $40,0 \%$ & $60,0 \%$ \\
\hline $\begin{array}{l}\text { Plus de } \\
\text { 1000 USD }\end{array}$ & $50,0 \%$ & $50,0 \%$ & $50,0 \%$ & $50,0 \%$ & - & - & $100 \%$ \\
\hline
\end{tabular}

\begin{tabular}{|c|c|c|c|c|c|c|c|c|}
\hline \multirow[b]{2}{*}{$\begin{array}{c}\text { Contribu- } \\
\text { tion }\end{array}$} & \multicolumn{5}{|c|}{ Domaine } & \multicolumn{3}{|c|}{ Expérience } \\
\hline & TV & Radio & $\begin{array}{c}\text { Jour- } \\
\text { naux } \\
\text { et Maga- } \\
\text { zines }\end{array}$ & $\begin{array}{l}\text { Web } \\
\text { Jour. }\end{array}$ & $\begin{array}{l}\text { Jour. } \\
\text { Free- } \\
\text { Lance }\end{array}$ & $\begin{array}{c}\text { Moins } \\
\text { que } \\
5 \text { ans }\end{array}$ & $\begin{array}{c}\text { Entre } \\
5 \text { et } 10 \\
\text { ans }\end{array}$ & $\begin{array}{l}\text { Plus } \\
\text { que } 10 \\
\text { ans }\end{array}$ \\
\hline O USD & $100.0 \%$ & - & - & - & - & $66.7 \%$ & - & $33.3 \%$ \\
\hline $\begin{array}{l}{[0-25} \\
\text { USD] }\end{array}$ & $66.7 \%$ & - & $16.7 \%$ & - & $16.7 \%$ & $66.7 \%$ & $33.3 \%$ & - \\
\hline $\begin{array}{l}\text { [26-100 } \\
\text { USD] }\end{array}$ & $66.7 \%$ & $11.1 \%$ & - & $11.1 \%$ & $11.1 \%$ & $60.0 \%$ & $20.0 \%$ & $20.0 \%$ \\
\hline $\begin{array}{l}\text { [101-250 } \\
\text { USD] }\end{array}$ & $75.0 \%$ & - & $25.0 \%$ & - & - & $28.6 \%$ & $14.3 \%$ & $57.1 \%$ \\
\hline $\begin{array}{l}\text { [251-500 } \\
\text { USD] }\end{array}$ & $16.7 \%$ & $16.7 \%$ & - & $33.3 \%$ & $33.3 \%$ & $100.0 \%$ & - & - \\
\hline $\begin{array}{l}\text { [501-1000 } \\
\text { USD] }\end{array}$ & $66.7 \%$ & $33.3 \%$ & - & - & - & - & $50.0 \%$ & $50.0 \%$ \\
\hline $\begin{array}{l}\text { Plus de } \\
1000 \text { USD }\end{array}$ & $75.0 \%$ & - & - & - & $25.0 \%$ & - & - & - \\
\hline
\end{tabular}


La ventilation de la propension à contribuer par données socio-économiques montre que ceux qui sont prêts à contribuer entre 26 et 100 USD sont pour une grande partie des femmes (80\%), des jeunes âgés entre 21 et 25 ans (60\%), actuellement travaillant en journalisme (60\%), surtout dans la télévision (66.7\%) et ayant une expérience récente de moins de 5 ans (60\%).

Ce profilage permet de donner de l'espoir concernant l'avenir des modes de financement des projets journalistiques qui compterait sur l'engagement d’une génération plus éclairée sur les nouveaux modèles économiques alternatifs.

\section{Conclusion}

Les déboires financiers des groupes médiatiques poussent à mettre en place de nouveaux modes de financement alternatifs qui permettraient d'assurer la pérennité des institutions médiatiques. Le financement participatif vient compléter les pratiques de journalisme participatif. Comme il est évident de faire appel au public pour lapprovisionnement en contenu, il est normal de lui faire appel pour l'approvisionnement en fonds. Comme le financement participatif a prouvé son efficacité dans le financement de projets artistiques, il n'est pas inenvisageable qu'il offre une planche de secours au naufrage financier des journaux et des fournisseurs d'information.

Lattitude des principaux concernés par ces évolutions au Liban savère nécessaire étant donné l'urgence de trouver des solutions pratiques et immédiates vu le nombre grandissant de medias déclarant leur faillite à cause du manque de financement. Le profil d'un potentiel militant pour le crowdfunding aurait le trait d'un jeune, ayant une expérience récente dans le domaine, travaillant surtout dans le domaine de la télévision et ayant une certaine familiarité et expérience avec les nouvelles plateformes de financement. Cependant, s'il sengage dans cette voie de financement, sa contribution resterait faible et tendrait vers un financement de médias éclatés, obéissant à un modèle économique plus dépouillé.

$\mathrm{Au}$-delà de la discussion matérielle de financement, il est impossible de discuter des modèles économiques du journalisme sans évoquer l'ingérence des partis et des personnalités politiques dans le financement de journaux. Le déclin financier actuel serait-il un signe de l'assèchement de la manne financière des groupes politiques ? Un appel au sursaut financier permettrait-il en retour de cesser la mainmise politique sur la liberté de la presse et permettra de libérer la parole et de rendre plus transparent et plus efficace la mission des journalistes? 


\section{Références}

AGRAWAL, A. ; CATALINI, C. ; GOLDFARB, A. Entrepreneurial finance and the flat-world hypothesis: evidence from crowd-funding entrepreneurs in the arts, 10(08), 2009.

AITAMURTO, T. The impact of crowdfunding on journalism: Case study of Spot. Us, a platform for community-funded reporting. Journalism practice, 5(4), 429-445, 2011.

BELLEFLAMME, P. ; LAMBERT, T. ; SCHWIENBACHER, A. Tapping the Right Crowd. Journal of Business Venturing. 29(5), 585-609, 2012.

BERTRAND, C. ; JAKUBOWSKI, B. Le fric, c'est chic: panorama du crowdfunding en 2016. In Annales des Mines-Réalités industrielles, 1, 38-43. FFE., 2016.

CAGE, J. Sauver les médias. Capitalisme, financement participatif et démocratie. Le Seuil, 2015.

CARVAJAL, M. ; GARCIA-AVILES, J.A. ; GONZALEZ, J.L. Crowdfunding and non-profit media: The emergence of new models for public interest journalism. Journalism Practice, 6(5-6), 638-647, 2012.

DE BUYSERE, K. ; GAJDA, O. ; KLEVERLAAN, R. ; MAROM, D. A Framework for European Crowdfunding [Online] Disponible sur: http://evpa.eu.com/wpcontent/uploads/2010/11, 2012 [consulté le 24 janvier 2013]

GERBER, E. ; M., HUI, J.S. ; KUO, P.Y. (2012). Crowdfunding: Why people are motivated to post and fund projects on crowdfunding platforms. Proceedings of the International Workshop on Design, Influence, and Social Technologies: Techniques, Impacts and Ethics, 2012.

HEKMAN, E. ; BRUSSEE, R. Crowdfunding and online social networks. 2nd Consortium on Applied Research and Professional Education. Manchester, UK. Utrecht, Netherlands: CARPE (the Consortium on Applied Research and Professional Education), 2013.

KUPPUSWAMY, V. ; BAYUS, B.L. Crowdfunding creative ideas: The dynamics of project backers in Kickstarter. UNC Kenan-Flagler Research Paper,15, 2015.

MOISSEYEV, A. Effect of social media on crowdfunding project results. Thèse défendue à l'Université du Nebraska, 2013.

MOLLICK, E. The Dynamics of Crowdfunding: Determinants of Success: An Explanatory Study. University of Pennsylvania - Wharton School. Journal of Business 2013. 
ONNEE, S. ; RENAULT, S. Le financement participatif: atouts, risques et conditions de succès. Gestion, 38(3), 54-65, 2013.

SÁNCHEZ-GONZÁLEZ, M. ; PALOMO-TORRES, M.B. Knowledge and Assessment of Crowdfunding in Communication: the View of Journalists and Future Journalists. Comunicar: Revista Científica de Comunicación y Educación, 22(43), 101-110, 2014.

SCHIFFRIN, A. Largent et les mots. La fabrique éditions, 2010. 